\title{
Management of type A dissection with malperfusion
}

\author{
Bo Yang, Himanshu J. Patel, David M. Williams, Narasimham L. Dasika, G. Michael Deeb \\ University of Michigan Medical Center, Ann Arbor, Michigan, USA \\ Correspondence to: Michael Deeb, MD. 1500 E Medical Ctr Dr, CVC 5147, Ann Arbor, MI 48109-5864, USA. Email: mdeeb@umich.edu.
}

\begin{abstract}
Malperfusion is a common lethal complication of acute aortic dissection following rupture, for which the optimal management strategy has yet to be clearly established. The objective of this study was to reassess the management of acute type A aortic dissection (Type A-AAD) with malperfusion. We retrospectively analyzed the outcomes of all patients with Type A-AAD with malperfusion at the University of Michigan and compared the results from patients that directly underwent open surgical repair versus those who had percutaneous reperfusion prior to open surgical repair. Based on the results, we developed a patient care protocol for the treatment of all patients with acute type A dissection. We later re-analyzed the long-term outcomes for patients using the protocol. The present study demonstrated that, although the outcomes for patients with acute type A aortic dissection with malperfusion syndrome treated with initial percutaneous reperfusion and delayed open surgical intervention are not as good as the results for patients with uncomplicated Type A-AAD that undergo immediate surgical repair, their outcomes continue the longterm outcomes of the former group are superior. To outdo patients with acute type A aortic dissection with malperfusion syndrome treated with immediate open surgical intervention. In conclusion, at the University of Michigan we continue to use our patient care protocol to treat patients with Type A-AAD.
\end{abstract}

Keywords: Malperfusion syndrome; fenestration and stenting; acute type A aortic dissection

Submitted Feb 04, 2016. Accepted for publication Jun 10, 2016.

doi: $10.21037 /$ acs.2016.07.04

View this article at: http://dx.doi.org/10.21037/acs.2016.07.04

\section{Introduction}

Malperfusion is the second most common lethal complication of acute aortic dissection following rupture. The occurrence varies in the literature anywhere from $10-33 \%$ and can occur with both acute type A and type B dissection (1). The early diagnosis and characterization of the extent of malperfusion is essential in determining the initial modality of therapy for the patient. Patients who are at the highest risk of fatality from malperfusion are those with delayed diagnosis of dissection or delayed presentation to a medical center equipped to handle acute aortic dissection. Longer time intervals between the actual episode of acute aortic dissection and subsequent diagnosis with treatment will result in a higher probability of irreversible endorgan ischemia with death of the patient. Patients who present with end-organ malfunction due to acute ischemia need immediate reperfusion with subsequent metabolic stabilization for the highest likelihood of survival (2).

\section{Etiology \& definition}

Malperfusion is defined as the loss of blood supply to a vital organ caused by branch arterial obstruction secondary to the dissection. When end-organ ischemia from ongoing branch arterial obstruction is not corrected in a timely manner, it can progress to end-organ malfunction with infarction, which is known as malperfusion syndrome. There is a distinct difference in the medical status, procedure choice and procedural outcomes of patients with malperfusion versus those with malperfusion syndrome and therefore it is imperative that the patient is diagnosed correctly and treated appropriately.

The obstruction of aortic branches can be classified as either dynamic, static or both depending on the pattern of anatomic obstruction, which can result in intermittent or persistent malperfusion of the organs (Figure 1) (4-6).

Dynamic obstruction is secondary to the motion of the intimal flap within the aortic lumen resulting from 


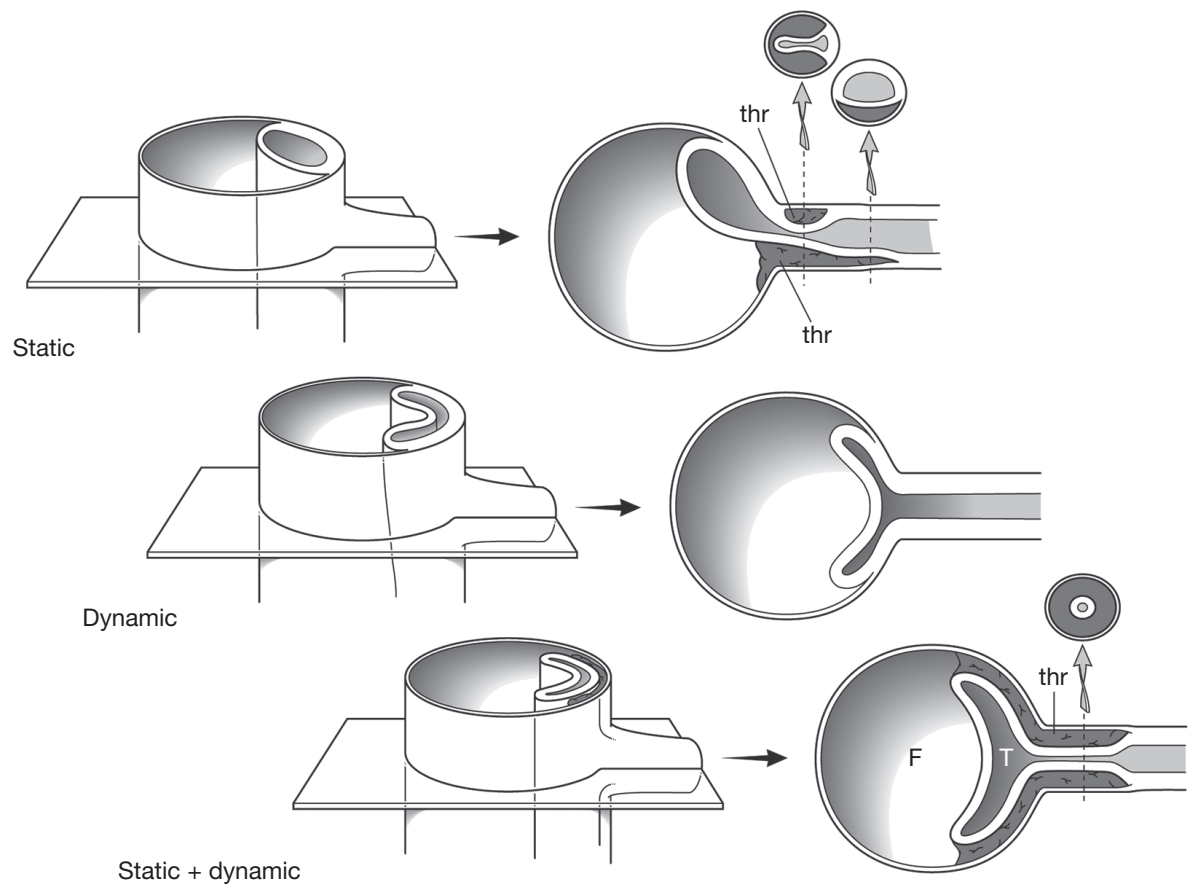

Figure 1 Diagram depicting anatomical relationship of dissection flap with branch artery origins, modified with permission from (3). In static obstruction, the dissection flap enters a branch artery's origin. If the false lumen reenters the branch artery distally, no pressure deficit may be present. If the reentry tear is small or absent (in which case the false lumen may thrombose), a pressure deficit is likely present. In dynamic obstruction, the dissection flap prolapses over the vessel origin like a curtain.

hemodynamic forces. The intimal flap may drape over and obstruct the orifice of a branch vessel to a vital organ, causing ischemia with potential end-organ dysfunction and infarction. As a dynamic phenomenon, malperfusion may vary depending on changes in blood pressure and hemodynamic forces, and in many cases can be adequately managed with medical therapy by controlling the blood pressure and hemodynamics (Figure 2). If this fails, intervention is needed $(4,5)$.

Static malperfusion is defined as dissection of the branch vessel with obstruction of the true lumen. In most cases this is secondary to thrombosis of the false lumen with compression and obstruction of the true lumen. This form of obstruction cannot be corrected with medical management and will require some form of interventional correction $(3,4)$.

Patients can present with either dynamic (Figure $3 A$ ), static or a combination of both forms (Figure $3 B$ ) of malperfusion and that is why it is essential to differentiate the status of malperfusion with angiography prior to determining the modality of definitive therapy (5).

\section{Incidence \& diagnosis}

Approximately one-third of patients with acute type A aortic dissection develop malperfusion (1). The diagnosis of malperfusion can be made with a combination of patient history, physical examination, laboratory values and imaging findings. The most common presentation for acute type A aortic dissection is excruciating chest pain radiating to the back. Unique to dissection is the fact that this pain may not be confined to the chest but may also radiate to the neck, abdomen, pelvis and extremities. Persistent pain in any of these other body areas should alert the care provider to the possibility of branch vessel occlusion with malperfusion and end-organ ischemia (malperfusion syndrome).

Physical exam can support the diagnosis of malperfusion. Absence of peripheral pulses should alert the care provider of the likelihood of malperfusion. In addition, abdominal tenderness, guarding and/or rebound tenderness as well as flank tenderness should alert the examiner of potential mesenteric ischemia. Any new motor or sensory deficit of an extremity is also characteristic of malperfusion syndrome of the extremities. Obviously any new neurologic deficit should 

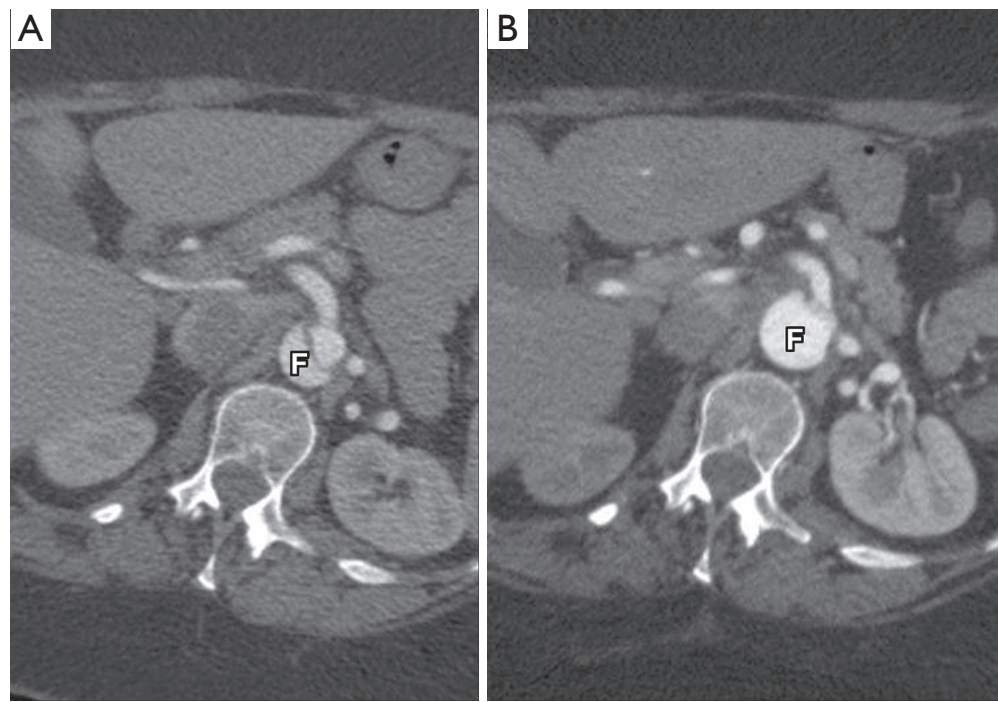

Figure 2 Baseline CT (A) at the level of the SMA shows a small but competent true lumen anteriorly with unobstructed SMA and symmetrically perfused kidneys. Blood pressure measured by an arm cuff was 96/64. One week later, when blood pressure was $188 / 73$, the patient complained of severe abdominal pain; and CT (B) now shows collapsed true lumen with reciprocal enlargement of the false lumen [F], and right renal hypoperfusion. At the time of angiographic evaluation, SMA trunk pressure was $42 / 38 \mathrm{mmHg}$. These images and corresponding blood pressures indicate the importance of sustained blood pressure control in patients with dissection anatomy predisposed to dynamic obstruction.
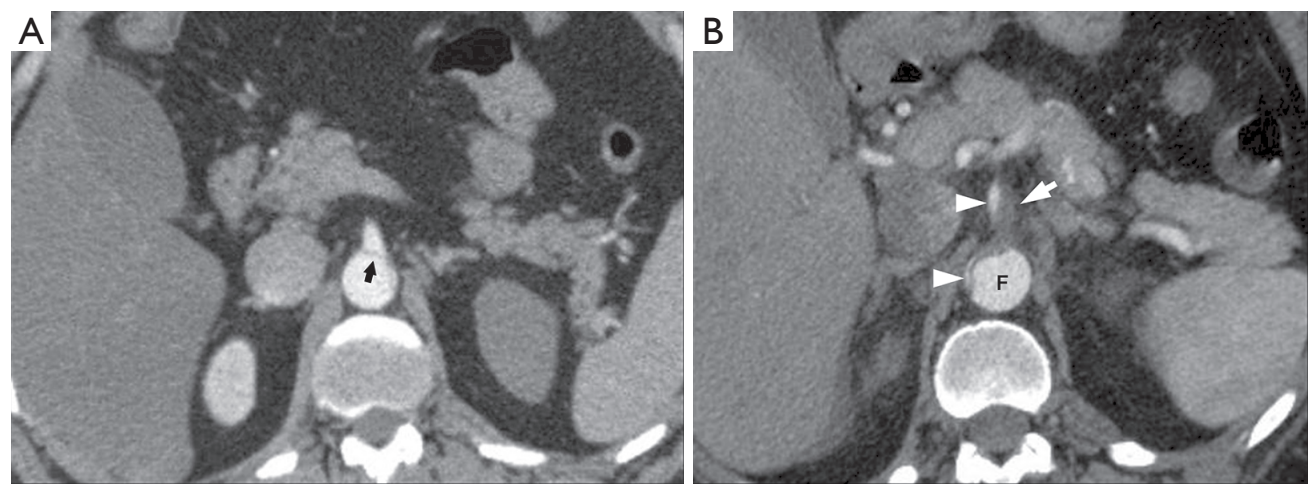

Figure 3 Forms of malperfusion. (A) CT at the level of the SMA origin shows single aortic lumen, which can be identified as the false lumen by carefully following it back to the heart on other slices (not shown). The left kidney is less well-opacified than the right. The dissection flap, consisting of the intima and portion of the media from the back wall of the aorta, is the thin line at the SMA origin (arrow) and is the cause of (dynamic) obstruction. Perfusion pressure in this SMA was $42 / 38 \mathrm{mmHg}$, which indicates that simple opacification of an artery does not rule out dynamic obstruction. Perfusion pressures in the aortic root true lumen and aortic false lumen at the T12 level were $91 / 64$ and 91/62 mmHg, respectively. In a second patient (B), CT at the level of the SMA origin shows that the true lumen of the aorta and SMA have been reduced to slit-like channels (arrowheads). The dissection flap enters the SMA trunk, and a small portion of the SMA true lumen seen here (anterior arrowhead) disappears distally. The SMA false lumen (arrow) occupies 2/3 of the vessel at this level, and is totally occlusive distally. Distal to the dissection in the SMA, perfusion pressure was 58/44 mmHg, compared to $118 / 56$ in the proximal aorta. 

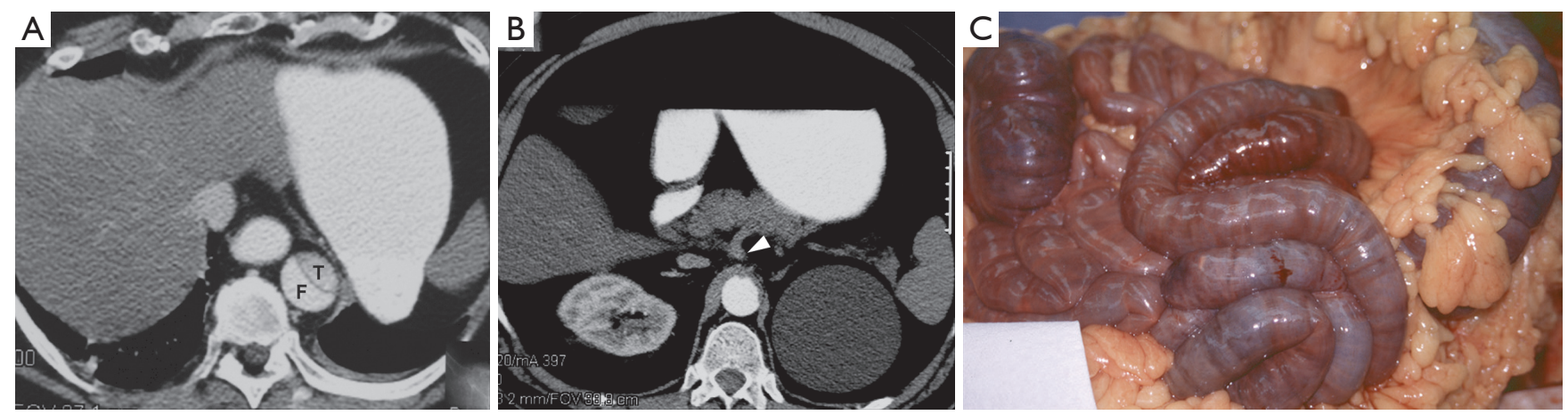

Figure 4 In a 48-year-old man with acute aortic dissection, CT image at the level of the distal descending aorta (A) shows a posterior false lumen $[\mathrm{F}]$ and a competent partially collapsed anterior true lumen $[\mathrm{T}]$. As the true and false lumens are traced from slice to slice from the chest (A) to the abdomen (B), the true lumen has collapsed completely and the false lumen now occupies nearly the entire aortic crosssection. Both celiac and SMA origins were covered by the dissection flap, which has collapsed up against the front wall of the aorta. The patient arrested and died in the Emergency Department before he could be transported to the angiography suite. At necropsy (C), the celiac and SMA were undissected, but the entire small bowel was infarcted.

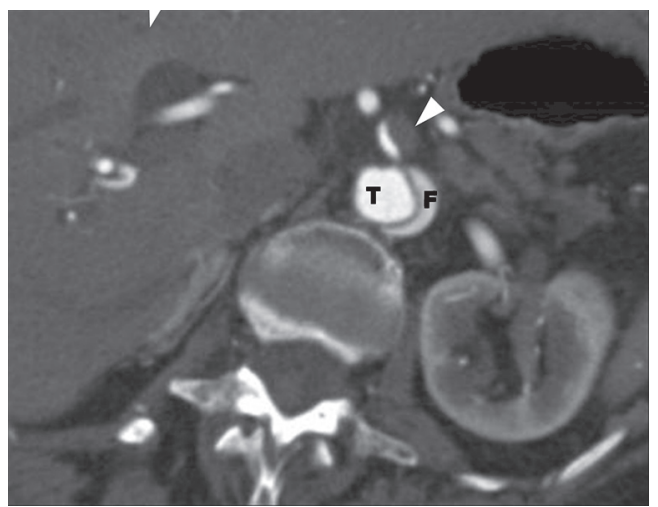

Figure 5 Abdominal CT just below the level of the SMA origin. The aortic true lumen ( $\mathrm{T})$ is uncompromised. The sagittal dissection flap extends into the SMA trunk without a distal reentry tear. The aortic false lumen (F) is patent, but its extension into the SMA has thrombosed and distally completely occludes the SMA (not shown). Distal SMA pressure was $43 / 38 \mathrm{~mm} \mathrm{Hg}$, compared to $129 / 43$ in the aortic root.

alert the examiner of the possibility of neurologic ischemia.

Laboratory result abnormalities are supportive and characteristic of malperfusion syndrome. A base deficit with metabolic acidosis, elevation of serum lactate levels, liver function tests, amylase and lipase levels, blood urea nitrogen and creatinine levels, myoglobin, total creatinine kinase and clotting indicators (prothrombin time and international normalized ratio) are all evidence for endorgan malperfusion.
Imaging studies such as gated aortic protocol computed tomography (CT) scans are diagnostic for aortic dissection and can lend evidence for the diagnosis of malperfusion. The sudden abrupt loss on CT scan of the aortic double lumen in an acute type A dissection at the level of the diaphragm (Figure 4) should alert the viewer that the true lumen has been eliminated, with the flap being pushed flush with the wall of the aorta causing obstruction of flow to vital branch vessels and dynamic malperfusion. Likewise, continuation of dual lumen patency of the aorta but with absence of flow in a branch vessel is evidence for static malperfusion (Figure 5).

Definitive diagnosis of malperfusion is made using angiography augmented with intravascular ultrasound and manometry $(3,4)$. During angiography, if dynamic malperfusion is diagnosed by manometry and ultrasound observation of the dissection flap, and is subsequently reversed with blood pressure control, then once metabolic stabilization occurs, definitive open surgical repair can be safely accomplished. If the anatomic picture with intravascular techniques documents dynamic malperfusion, but blood pressure control cannot correct the malperfusion, or if the study documents isolated static vascular occlusion then correction can be immediately performed using intravascular fenestration and stenting of the true lumen (2-6).

\section{Treatment techniques}

If the malperfusion is dynamic, and can be corrected by 
A

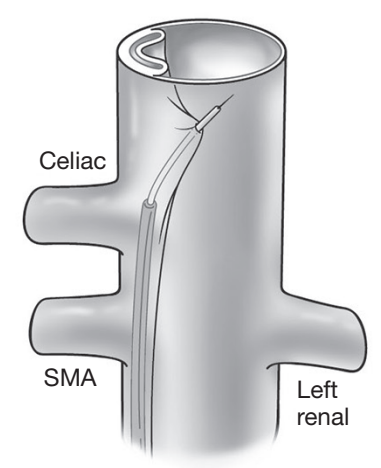

B

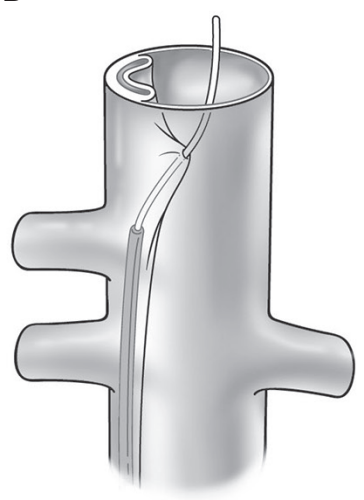

B

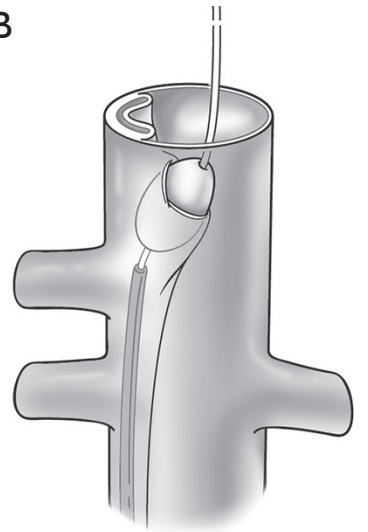

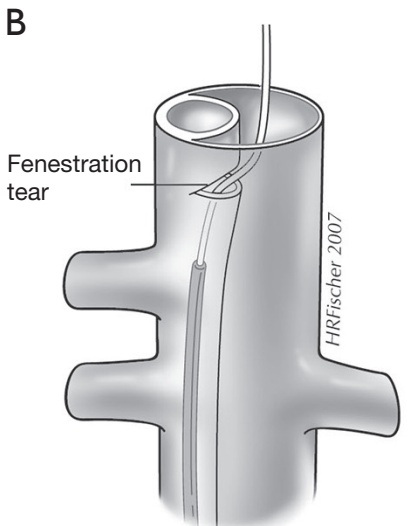

Figure 6 Diagram showing steps in fenestration. In (A), a needle is advanced across the dissection flap, based on IVUS guidance or CT knowledge of the true and false lumen orientation. After the needle and catheter have crossed the dissection flap, a guidewire is advanced (B), and a $16 \mathrm{~mm}$ balloon is centered on the flap and dilated (C). The balloon is then deflated completely and retracted (D), leaving a transverse tear on the flap. Before placement of a stent in the true lumen, the wire is withdrawn across the flap and re-advanced in the true lumen. Reprinted with permission from Journal of Thoracic and Cardiovascular Surgery (8).

blood pressure control, then timely open surgery with restoration of true aortic luminal flow by eliminating the tear and replacing the ascending aorta will often correct the secondary malperfusion $(5,7)$. With static malperfusion, delayed diagnosis or delayed treatment of dissection, however, established end-organ ischemia and clinical deterioration are typical, and the conventional strategy of immediate ascending aortic repair may be suboptimal. In these situations, alternative management strategies to immediate open surgery should be considered (2-4).

Intravascular fenestration and true lumen stenting is a quick technique of resolving malperfusion due to dynamic obstruction and reestablishing immediate blood flow to the ischemic organs without the delay, trauma burden, upregulation of inflammatory factors from cardiopulmonary bypass and coagulopathy with large transfusions of blood products secondary to immediate open heart surgery in a patient with severe metabolic end-organ dysfunction (2-4).

Intravascular access for fenestration and stenting is obtained through the femoral vessels with percutaneous needle puncture and the introduction of a sheath over a guide wire into the true lumen of the aorta. CT imaging, intravascular ultrasound and angiography can be used as aids to successfully gain access into the true lumen. During the fenestration procedure, a needle is introduced through the sheath and used to pierce the aortic flap to gain access across the flap and advance the catheter into the false lumen. Once access is gained across the flap, the needle is replaced with a guidewire and a balloon catheter is introduced across the flap over the wire and positioned within the tear in the flap caused by the knife. The balloon is inflated, deflated and withdrawn back, creating a large hole in the flap to allow free flow of blood across the flap and equilibration of pressures between the two lumens (Figure 6). A 16-mm self-expanding stent is then placed in the true lumen of the aorta just above the tear to help maintain aortic true lumen patency (Figure 7). After hemodynamic malperfusion has been corrected, it is imperative to rule out simultaneous static malperfusion which would require additional intravascular branch graft stenting to resolve all the malperfusion $(3,4,8-10)$ (Figure 8). Persistent malperfusion can be confirmed by measuring the blood pressure gradient between the dissected aortic branch and the ascending aorta.

For acute type A patients, once this procedure is completed with successful reperfusion of the ischemic organs, the patient should be allowed to stabilize from ischemia-reperfusion injury with metabolic correction prior to the definitive open surgical procedure. This will allow sufficient time to assess the extent of end-organ damage and perform any necessary ancillary procedures, such as exploratory laparotomy and resection of dead organs for end-stage mesenteric ischemia or fasciotomy and debridement of muscle tissue for limb ischemia, to establish metabolic stabilization prior to definitive open corrective procedure of the aorta. This stabilization period has varied in our experience from 24 hours to several months $(2,5,6)$. 


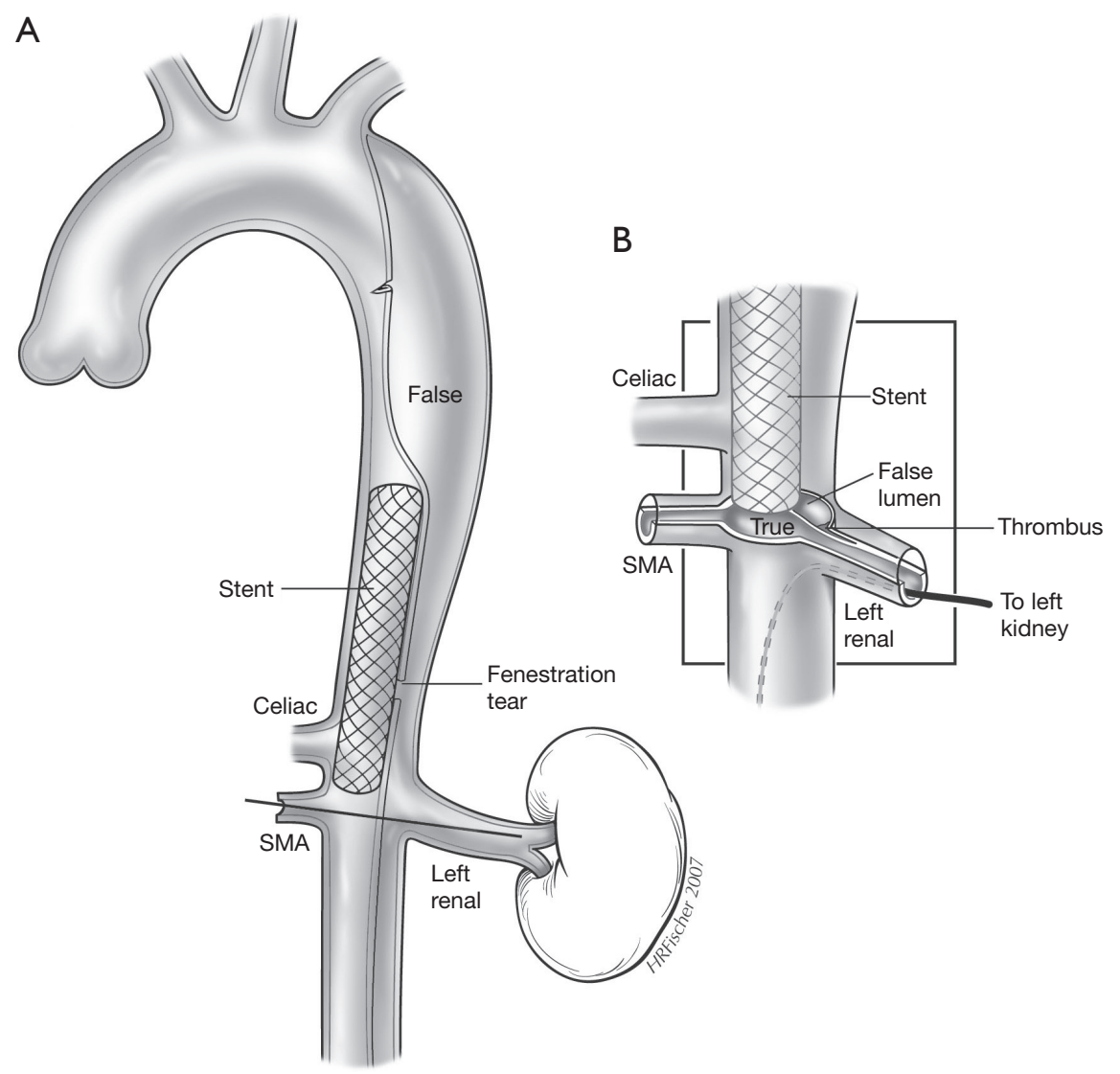

Figure 7 If the true lumen does not decompress after the fenestration tear is created (observed by IVUS and confirmed by normalization of perfusion pressures), then a large-diameter self-expanding stent is deployed exclusively in the aortic true lumen. We deploy the stent so as to land at the rostral margin of the SMA. The stent lies across the fenestration tear as well as the celiac origin, but neither hemolysis nor functional celiac stenosis has been a problem in clinical follow-up. Reprinted with permission from ref 3 .

\section{Results of treatment}

\section{Acute type A dissection}

In 1997, we described our early results for the strategy of operative delay for those patients presenting with acute type A dissection, malperfusion, and ischemic endorgan dysfunction (2). In that study, a historical cohort of patients presenting with ischemic end-organ dysfunction from malperfusion taken directly for open repair was compared with a cohort managed with initial percutaneous fenestration with aortic stenting, selective branch vessel stenting, and delayed operative repair after resolution of the perfusion injury. The in-hospital mortality of $89 \%$ for the historical group was statistically significantly higher than those patients who underwent operative delay at an overall $25 \%$ mortality $(\mathrm{P}<0.01)$, which consisted of $15 \%$ mortality from ascending aortic rupture with tamponade and $10 \%$ incidence of death due to complications of end organ ischemia.

These early results led to a consistent strategy of care for all patients presenting at our institution with acute type A aortic dissection and malperfusion with end-organ ischemic dysfunction (6). This strategy consisted of percutaneously restoring end-organ perfusion and obtaining metabolic stability prior to open surgical aortic reconstruction (Figure 9). After 10 years, we reviewed the clinical outcomes to determine if we had long-term data-driven validity for the adoption of this strategy.

Over that time period, 196 patients with acute type A dissection presented to the University of Michigan Hospital, and 70 were diagnosed with malperfusion and end-organ dysfunction (malperfusion syndrome). These 70 patients underwent percutaneous end-organ reperfusion with medical stabilization, followed by delayed 

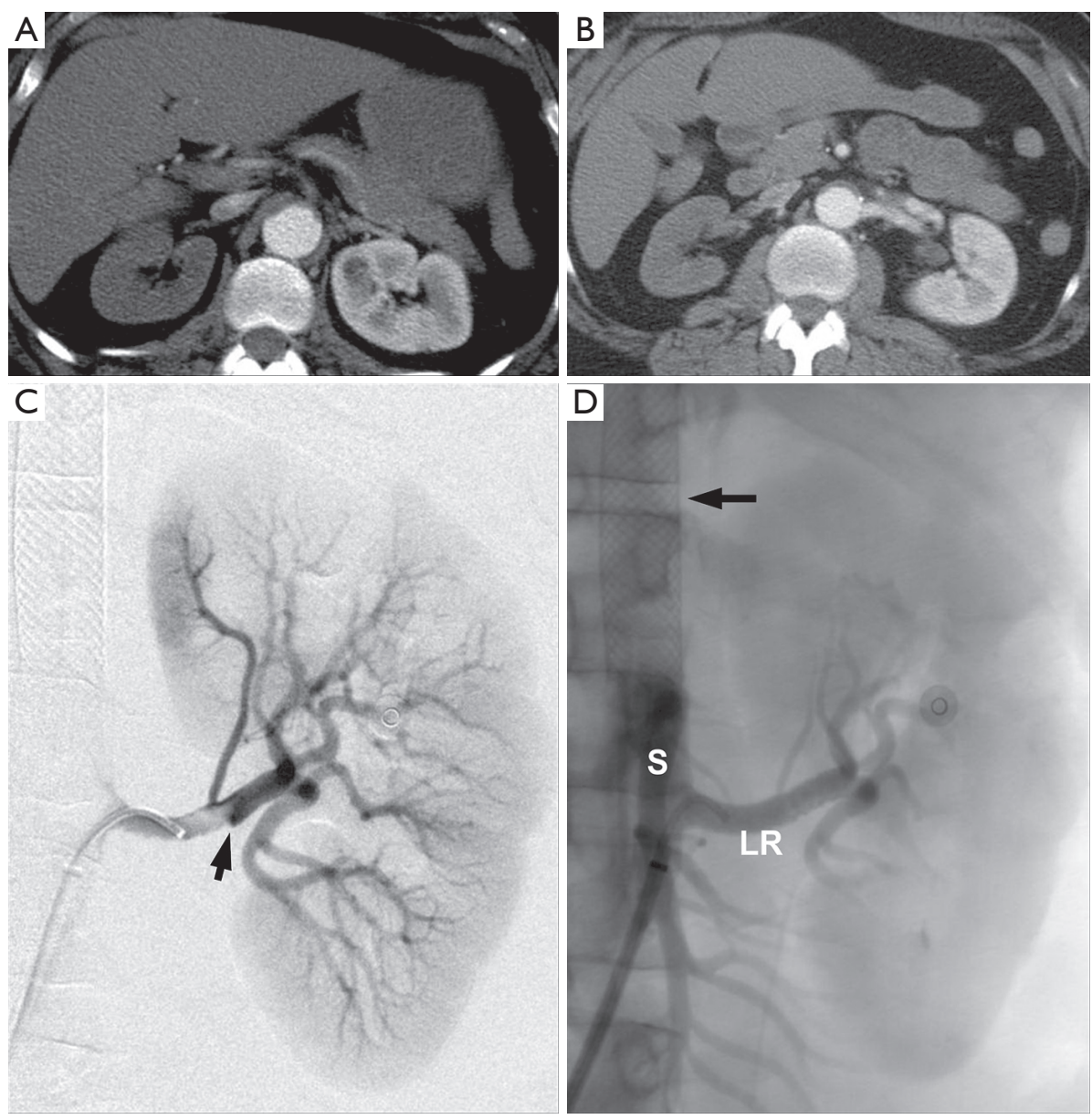

Figure 8 CT at the level of the SMA (A) shows the false lumen completely occupying the aorta cross-section, with the true lumen collapsed against the anterior aortic wall, occluding the SMA and right renal artery (not seen at this level). The right kidney is relatively hypoperfused. At a lower level (B), the left renal artery is seen to arise from the aortic false lumen. At angiographic evaluation, gut and right renal perfusion were compromised by dynamic obstruction (perfusion pressures 29/26 and 55/37, respectively) and the left by static obstruction (perfusion pressure 82/44, measured at the renal hilum), with aortic root pressure 112/53. Baseline left renal arteriogram (C) shows dissection reentry (arrow) just proximal to the hilum. After treatment (D) including fenestration, aortic stenting (arrow), and left renal artery stenting, all three branch artery perfusion pressures were raised to systemic levels. S, superior mesenteric artery; LR, left renal artery.

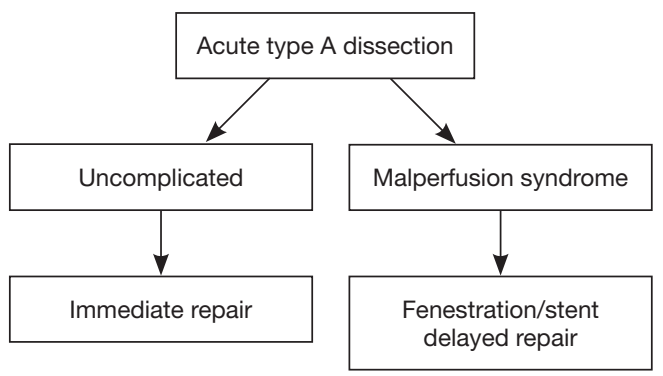

Figure 9 Flow diagram for management of acute type A dissection at the University of Michigan. open surgical correction (Figure 10). There was a $95 \%$ success rate in opening the obstructed vessels using the percutaneous technique. Twenty-three of the 70 patients $(38 \%)$ treated with the percutaneous technique died prior to surgical repair, $12(19 \%)$ died of aortic rupture and $11(19 \%)$ from complications of end organ damage from malperfusion. Forty-seven patients $(62 \%)$ recovered and underwent delayed open surgical repair (Figure 11). This was in stark contrast with the 126 patients who did not have malperfusion or end-organ dysfunction, whose early operative mortality was $9.5 \%$ and median survival was 


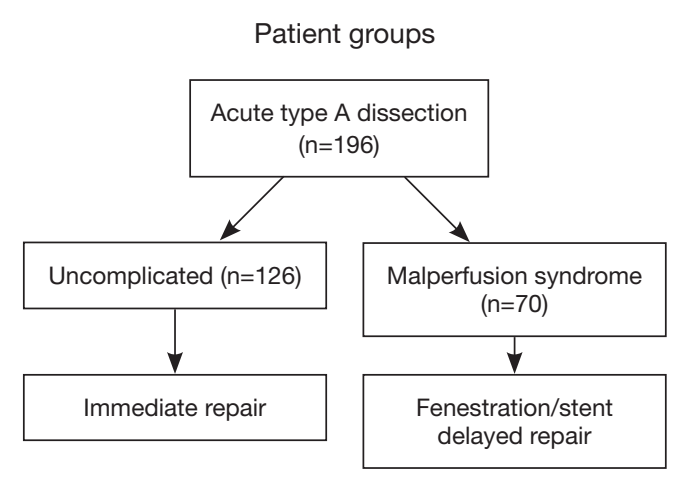

Figure 10 Flow pattern of patients in the study with acute type A d.

Early outcomes-malperfusion syndrome

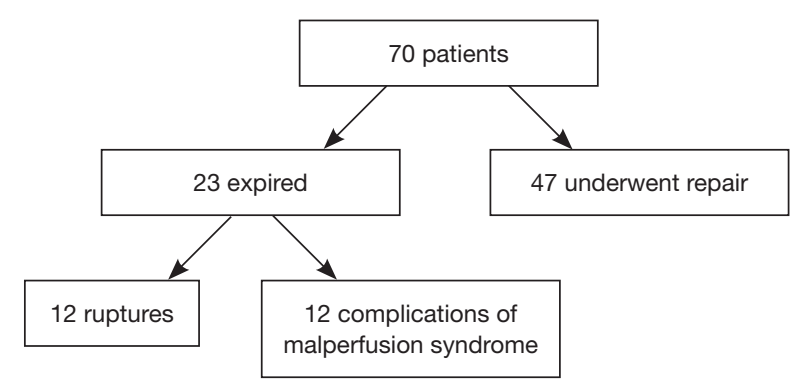

Figure 11 Outcomes for malperfusion syndrome patients with percutaneous fenestration and stenting.

96 months, whereas the median survival was 54 months for the patients with malperfusion syndrome $(\mathrm{P}<0.001)$ (Figure 12) (6). This data supports the notion that the complication of malperfusion with end-organ dysfunction carries a high mortality risk irrespective of early reperfusion, stabilization and delayed surgical reconstruction. However, these results for the malperfusion syndrome group were still statistically superior to the outcomes for the malperfusion syndrome patients directly undergoing surgical aortic reconstruction, strongly supporting our strategy with datadriven clinical outcomes (2).

We then analyzed the outcomes for the uncomplicated patients $(n=126)$ that directly underwent open surgical reconstruction versus the malperfusion syndrome patients who survived the percutaneous protocol and went on to open aortic reconstruction $(n=47)$. For this group of patients who survived the percutaneous protocol, the median delay from fenestration \pm stenting to open surgical repair was four days (Figure 13).

This analysis was to determine whether corrected

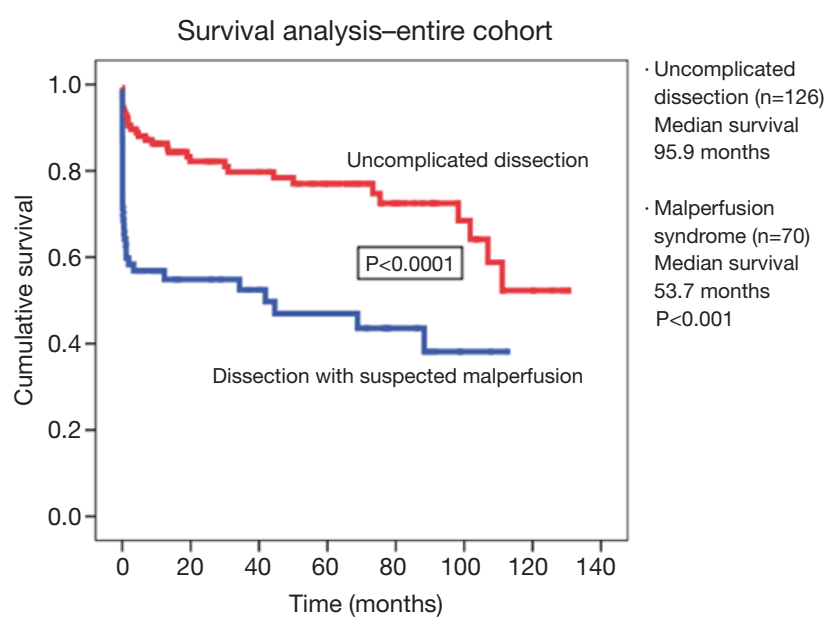

Figure 12 Graphic display comparing the survival of the 2 groups (Uncomplicated and Malperfusion Syndrome) presenting with acute type A dissection.

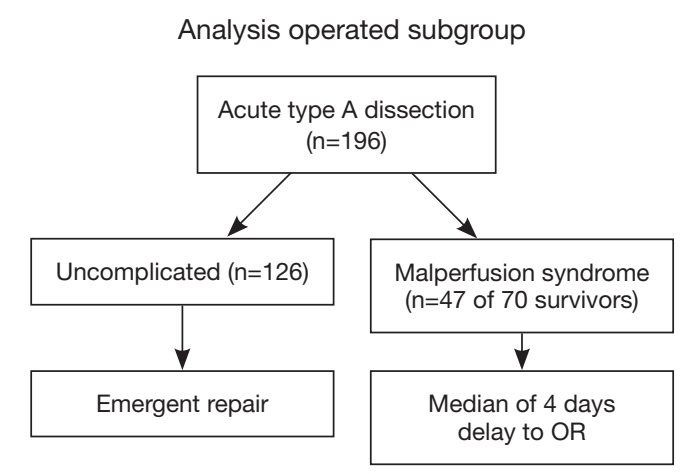

Figure 13 Flow diagram showing the median time of delay to open surgery for malperfusion syndrome patients.

malperfusion syndrome would have a negative adverse impact on the outcomes for delayed open surgical aortic reconstruction, once the syndrome was resolved. The analysis compared early outcomes for death, stroke, and need for dialysis or tracheostomy between the two groups and showed no statistically significant difference between the two groups for the open aortic reconstruction (Table 1). Cumulative survival curves also showed no statistical significance $(\mathrm{P}=0.45)$, with uncomplicated patients having a median survival of 96 months versus 81 months for the malperfused patients who proceeded to surgery (Figure 14).

A recent GERAADA analysis demonstrated that malperfusion is dependent on the number of affected systems (11). An analysis of our data was also undertaken 


\begin{tabular}{llll}
\multicolumn{4}{l}{ Table 1 Operated subgroup-morbidity } \\
\hline Morbidity $(\mathrm{n}=173)$ & UC (\%) & Operated MP (\%) & P value \\
\hline Death & 9.5 & 8.5 & 1.0 \\
Stroke & 7.9 & 4.3 & 0.52 \\
Dialysis & 8.7 & 19.1 & 0.07 \\
Trachestomy & 7.1 & 4.3 & 0.73 \\
\hline
\end{tabular}

This table shows the early comparisons of adverse outcomes between the uncomplicated patients undergoing immediate surgery (UC) and those patients with malperfusion syndrome that underwent delayed surgery (MP).

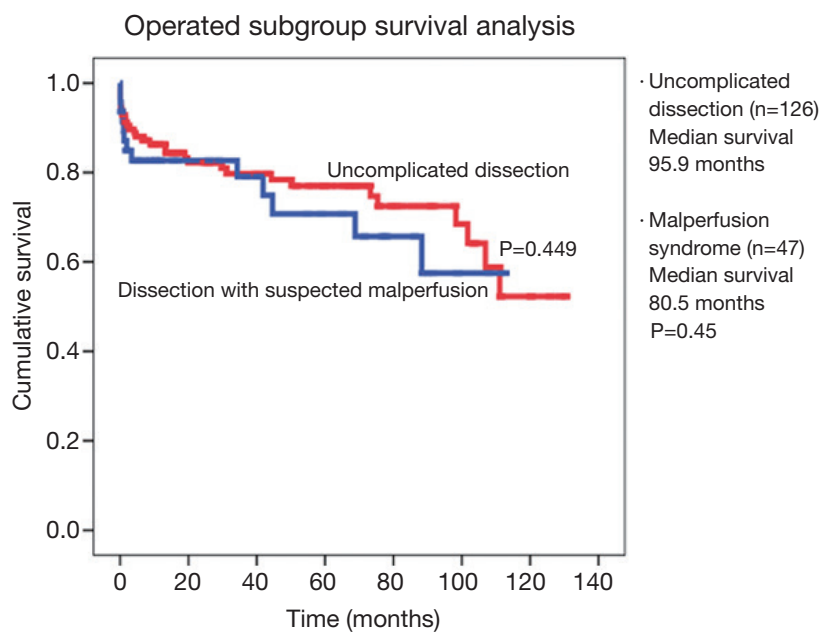

Figure 14 Graphic display comparing the survival of the delayed operative malperfusion patients to the uncomplicated patients who underwent immediate surgery.

to determine whether malperfusion of any particular vascular bed was an independent correlate of mortality and mesenteric malperfusion was a statistically significant independent predictor of mortality.

It was concluded from analysis of long-term data that the presence of acute type A dissection with malperfusion and end-organ dysfunction (malperfusion syndrome) is an important adverse factor for long-term survival, particularly in the setting of mesenteric malperfusion. The strategy of immediate reperfusion, stabilization, and planned open aortic reconstruction still carries a significant risk for early mortality (38\%). Those patients who do survive the initial malperfusion and undergo delayed open aortic reconstruction experience no adverse effect and have a similar operative and late survival when compared with those presenting with uncomplicated dissection. Despite the increased risk of malperfusion syndrome, the strategy of immediate percutaneous reperfusion with medical metabolic stabilization followed by delayed open surgical aortic reconstruction is a valid strategy supported by datadriven clinical outcomes (6).

We believe it is important that the distinction is clearly understood between malperfusion (ongoing arterial obstruction) and malperfusion syndrome (arterial obstruction with ischemic end-organ dysfunction). Those patients with malperfusion but no significant adverse endorgan effects (malperfusion syndrome) are best treated with immediate surgical repair. An acute type A dissection is still a surgical emergency, and patients without endorgan ischemia, either with or without malperfusion have an equal operative risk, which is significantly lower than patients with both malperfusion and end-organ dysfunction (5-7). Patients with malperfusion syndrome (end-organ dysfunction due to ischemia), however, should undergo fenestration with or without stenting to reperfuse the ischemic organs with stabilization prior to open surgical repair of the acute type A dissection. Figure 9 is a flow diagram for our current protocol for the treatment of patients with acute type A aortic dissection.

\section{Acknowledgements}

None.

\section{Footnote}

Conflicts of Interest: The authors have no conflicts of interest to declare.

\section{References}

1. Geirsson A, Szeto WY, Pochettino A, et al. Significance of malperfusion syndromes prior to contemporary surgical repair for acute type A dissection: outcomes and need for additional revascularizations. Eur J Cardiothorac Surg 2007;32:255-62.

2. Deeb GM, Williams DM, Bolling SF, et al. Surgical delay for acute type A dissection with malperfusion. Ann Thorac Surg 1997;64:1669-75; discussion 1675-7.

3. Williams DM, Lee DY, Hamilton BH, et al. The dissected aorta: percutaneous treatment of ischemic complications--principles and results. J Vasc Interv Radiol 1997;8:605-25.

4. Slonim SM, Miller DC, Mitchell RS, et al. Percutaneous 
balloon fenestration and stenting for life-threatening ischemic complications in patients with acute aortic dissection. J Thorac Cardiovasc Surg 1999;117:1118-26.

5. Deeb GM, Patel HJ, Williams DM. Treatment for malperfusion syndrome in acute type $\mathrm{A}$ and $\mathrm{B}$ aortic dissection: A long-term analysis. J Thorac Cardiovasc Surg 2010;140:S98-S100; discussion S142-S146.

6. Patel HJ, Williams DM, Dasika NL, et al. Operative delay for peripheral malperfusion syndrome in acute type A aortic dissection: a long-term analysis. J Thorac Cardiovasc Surg 2008;135:1288-95; discussion 1295-6.

7. Rampoldi V, Trimarchi S, Eagle KA, et al. Simple risk models to predict surgical mortality in acute type A aortic dissection: the International Registry of Acute Aortic Dissection score. Ann Thorac Surg 2007;83:55-61.

Cite this article as: Yang B, Patel HJ, Williams DM, Dasika NL, Deeb GM. Management of type A dissection with malperfusion. Ann Cardiothorac Surg 2016;5(4):265-274. doi:10.21037/acs.2016.07.04
8. Williams DM, Lee DY, Hamilton BH, et al. The dissected aorta: percutaneous treatment of ischemic complications--principles and results. J Vasc Interv Radiol 1997;8:605-25.

9. Dake MD, Kato N, Mitchell RS, et al. Endovascular stent-graft placement for the treatment of acute aortic dissection. N Engl J Med 1999;340:1546-52.

10. Williams DM, Lee DY, Hamilton BH, et al. The dissected aorta: part III. Anatomy and radiologic diagnosis of branch-vessel compromise. Radiology 1997;203:37-44.

11. Czerny M, Schoenhoff F, Etz C, et al. The Impact of PreOperative Malperfusion on Outcome in Acute Type A Aortic Dissection: Results From the GERAADA Registry. J Am Coll Cardiol 2015;65:2628-35. 